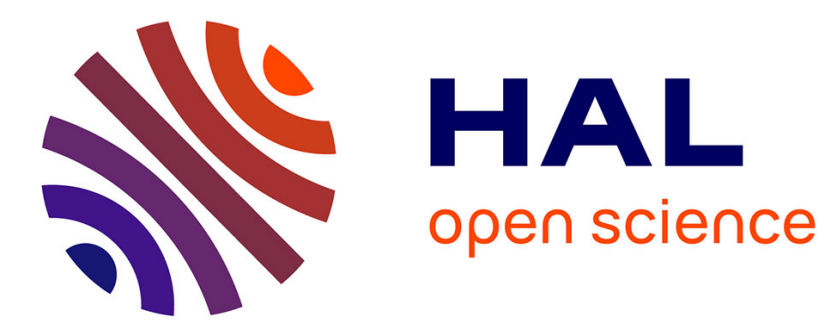

\title{
Conclusion générale. Gestion de la biodiversité, réalisations concrètes. \\ Jean-Claude Rameau
}

\section{To cite this version:}

Jean-Claude Rameau. Conclusion générale. Gestion de la biodiversité, réalisations concrètes.. Revue forestière française, 2001, 53 (sp), pp.315-317. 10.4267/2042/5318 . hal-03443504

\section{HAL Id: hal-03443504 https://hal.science/hal-03443504}

Submitted on 23 Nov 2021

HAL is a multi-disciplinary open access archive for the deposit and dissemination of scientific research documents, whether they are published or not. The documents may come from teaching and research institutions in France or abroad, or from public or private research centers.
L'archive ouverte pluridisciplinaire HAL, est destinée au dépôt et à la diffusion de documents scientifiques de niveau recherche, publiés ou non, émanant des établissements d'enseignement et de recherche français ou étrangers, des laboratoires publics ou privés. 


\section{CONCLUSIONS}

\section{CONCLUSION GÉNÉRALE}

\section{Jean-Claude Rameau}

Il est toujours difficile de proposer une conclusion à un colloque aussi riche par les idées et les points de vue exprimés. Nous insisterons plus particulièrement sur les évolutions très perceptibles qui se sont développées au cours de ces dix ans nous séparant du premier colloque "Patrimoines naturels forestiers".

Nous soulignerons déjà l'atmosphère très détendue qui a régné au cours de ces journées entre les divers acteurs, ce qui traduit de nouvelles habitudes : le développement de travaux menés en commun entraînant une meilleure connaissance réciproque ; la mise au point, en partenariat, d'une plate-forme commune en matière d'identification et de prise en compte de la diversité dans la gestion.

Comme partenariat, nous pouvons citer la généralisation des actions de Conservatoires botaniques nationaux au niveau de la préparation des aménagements forestiers, les actions menées entre l'Office national des Forêts (ONF) et Réserves naturelles de France sur les tourbières et leur gestion, les collaborations fructueuses qui s'établissent entre l'ONF et les Parcs naturels régionaux par le biais du volet forestier des chartes (collaboration exemplaire à souligner dans le Parc des Vosges du Nord, dans le cadre de la Réserve MAB Biosphère), les travaux menés par la Ligue pour la Protection des Oiseaux ou l'Office pour les Insectes, à la demande des gestionnaires de l'ONF.

Par ailleurs, la concertation, les réflexions en commun sur la gestion conservatoire se développent dans certains parc nationaux (Mercantour, Cévennes, Écrins...). Nous noterons également les bienfaits de certaines formations continues ouvertes à tous les gestionnaires d'espaces (organisées par l'Atelier technique des Espaces naturels, le Centre national de Formation forestière, l'École nationale du Génie rural, des Eaux et des Forêts...), avec des stages :

- sur la gestion forestière, la dynamique forestière, l'aménagement forestier en forêt ordinaire ;

- sur la gestion forestière dans les Parcs nationaux;

- sur Natura 2000 (identification et cartographie des habitats, réflexions en commun sur la gestion adaptée à préconiser...).

Ces stages entraînent une meilleure connaissance réciproque, non seulement des pratiques de gestion, de leur justification (compte tenu des milieux, de leur fonctionnement, de leur dynamique) mais aussi des objectifs et missions de chacun, ce qui contribue à aplanir certaines divergences souvent mal fondées...

Bien sûr, on constate encore des retards, des oublis, des erreurs chez les gestionnaires forestiers ; des progrès restent à faire. On observe encore des blocages dans le monde associatif, des réticences pour travailler avec les forestiers, pour les aider. On rencontre parfois de la mauvaise foi... un refus de reconnaître l'évolution évidente des pratiques au cours des quinze dernières années, ou la difficulté d'accepter le bien-fondé d'un compromis entre une certaine fonction 
économique de la forêt et la prise en compte des objectifs de conservation. Il est fondamental de poursuivre cette collaboration fructueuse, dans un objectif partagé, commun d'une meilleure conservation de la biodiversité.

Nous trouvons remarquable l'investissement parfois important de certains gestionnaires forestiers dans la connaissance des divers patrimoines naturels et dans l'expérimentation en matière de gestion. Nous souhaitons que les "chefs" permettent l'épanouissement de telles passions en les mettant au service de la gestion durable.

Du côté de Natura 2000, nous notons avec satisfaction une décrispation... et des travaux très intéressants conduits pour la connaissance et la gestion des habitats et des populations d'espèces. Nos différentes tournées sur le terrain nous révèlent :

- la nécessité de plus de travaux élaborés en commun (fabrication d’outils communs sur le plan régional pour l'identification des habitats) ;

- la nécessité, pour les experts, de compléter la panoplie des outils de vulgarisation pour venir en aide aux chargés d'étude et gestionnaires.

Nous tenons à souligner l'énorme travail de grande qualité mené par la communauté des généticiens dans le domaine de la conservation des ressources génétiques forestières. Nous avons noté avec satisfaction que nous disposerons, dans un futur proche, de recommandations pratiques à l'usage des gestionnaires.

Une bonne connaissance de la diversité passe par une coopération entre les spécialistes de diverses disciplines; nous voyons peu à peu se constituer des réseaux de chercheurs, qui ont pour objectif de mieux "balayer" les divers éléments de la biodiversité, leurs relations et les moyens les plus appropriés pour en assurer la pérennité.

Sur le plan entomologique, nous avons noté la nécessité de développer de nouveaux travaux et de sortir quelques recommandations pratiques.

De gros progrès sont réalisés dans la gestion des forêts tropicales :

- en Guyane avec la mise au point d'aménagements très élaborés, intégrant la conservation d'espaces non exploités ; reste à convaincre les exploitants, à les former... ;

- à La Réunion, nous avons vu l'importance du réseau de réserves biologiques et la conservation effective des types forestiers endémiques de l'île.

Nous avons attiré l'attention, à plusieurs reprises, sur le poids de l'histoire, dans la diversité actuelle. Ce poids est à mettre en évidence dans toutes les forêts. Ces réflexions ont permis d'insister sur le fait que nous devons également prendre en compte et protéger les patrimoines culturels au niveau des forêts (mégalithes, vestiges divers, patrimoines ethnologiques, historiques...).

Nous avons apprécié d'entendre nos collègues pastoralistes de l'Institut national de la Recherche agronomique (INRA) ; il est important pour les forestiers de connaître les approches développées, les conceptions dans les domaines pastoraux.

Un acquis important réside dans les résultats menés sur la Biodiversité forestière : cette biodiversité varie en un lieu précis, à travers les cycles forestiers. Cette réalité fait peu à peu son chemin et bat en brèche les visions trop statiques d'évaluation de la gestion élaborées par certaines structures, autorités (“suivi de Natura 2000"...). Il faut travailler à l'échelle de la forêt pour évaluer la qualité de la conservation des patrimoines et se focaliser sur la résilience des espèces, des structures... à travers les actions de gestion, à travers le temps. 
Nous prenons note de l'émergence de nouveaux problèmes : les menaces représentées par les plantes exotiques envahissantes dans certains milieux, en métropole, milieux présentant une faible maturité sylvigénétique (ripisylves avec Renouée du Japon, Budleya, Robinier...) ou dans certaines îles (Réunion, Tahiti...). Là encore, il est nécessaire d'expliquer, de convaincre, de ne pas utiliser certaines espèces dans les réaménagements paysagers et d'arriver au plus vite à l'établissement de stratégies efficaces (comme en Australie, aux îles Hawaï...).

Si de nombreux points positifs que nous venons de relever ont été présentés, nous nous devons d'éviter l'autosatisfaction. Il reste beaucoup à faire :

- nous n’avons pas vu présentées de stratégies générales, élaborées et mises en place avec, en particulier, les étapes importantes de l'évaluation, la définition de stratégies s'appuyant sur une hiérarchisation... ;

- nous n'avons rien entendu sur le suivi de la biodiversité ; des travaux sont indispensables, rapidement, dans ce domaine ;

- enfin, il faut vulgariser, porter à la connaissance pour favoriser le développement de la démarche patrimoniale.

Le travail est à parfaire dans les domaines de la communication, de la gestion, du faire connaître, $\mathrm{du}$ travail en partenariat, en matière de formation continue.

Il faudrait réfléchir à des stratégies à développer en forêt privée pour aider les experts, les gestionnaires dans ces domaines.

En une phrase, nous dirons que nous sommes très satisfaits, optimistes, mais il reste encore beaucoup à faire. 
